\title{
Long-term Outcomes of Additional Endoscopic Treatments for Patients with Positive Lateral Margins after Endoscopic Submucosal Dissection for Early Gastric Cancer
}

\author{
Tae-Se Kim, Byung-Hoon Min, Yang Won Min, Hyuk Lee, Poong-Lyul Rhee, Jae J. Kim, and Jun Haeng Lee \\ Department of Medicine, Samsung Medical Center, Sungkyunkwan University School of Medicine, Seoul, Korea
}

\author{
Article Info \\ Received May 6, 2021 \\ Revised July 4, 2021 \\ Accepted July 6, 2021 \\ Published online September 1, 2021

\section{Corresponding Author} \\ Byung-Hoon Min \\ ORCID https://orcid.org/0000-0001-8048-361X \\ E-mail bhmin@skku.edu \\ Jun Haeng Lee \\ ORCID https://orcid.org/0000-0002-5272-1841 \\ E-mail stomachlee@gmail.com
}

\begin{abstract}
Background/Aims: It is uncertain whether additional endoscopic treatment may be chosen over surgery in patients with positive lateral margins (pLMs) as the only non-curative factor after endoscopic submucosal dissection (ESD) for early gastric cancer (EGC). We aimed to compare the long-term outcomes of additional endoscopic treatments in such patients with those of surgery and elucidate the clinicopathological factors that could influence the treatment selection.

Methods: A total of 99 patients with 101 EGC lesions undergoing additional treatment after noncurative ESD with pLMs as the only non-curative factor were analyzed. Among them, 25 (27 lesions) underwent ESD, 29 (29 lesions) underwent argon plasma coagulation (APC), and 45 (45 lesions) underwent surgery. Clinicopathological characteristics and long-term outcomes were compared.

Results: Residual tumor was found in $73.6 \%$ of cases. The presence of multiple pLMs was associated with higher risk of residual tumor $(p=0.046)$. During a median follow-up of 58.9 months, recurrent or residual lesions after additional ESD and APC were found in $4 \%(1 / 25)$ and $6.8 \%$ (2/29) of patients, respectively. However, all were completely cured with surgery or repeated ESD. There were no extragastric recurrences after additional endoscopic treatment. Lymph node metastasis was identified after additional surgery in one (2.2\%) patient with an EGC showing histological heterogeneity.

Conclusions: Given the favorable long-term outcomes, additional ESD or APC may be an acceptable choice for patients with pLMs as the only non-curative factor after ESD for EGC. However, clincopathological characteristics such as multiple pLMs and histological heterogeneity should be considered in the treatment selection. (Gut Liver 2022;16:547-554)
\end{abstract}

Key Words: Endoscopic mucosal resection; Margins of excision; Outcomes; Stomach neoplasms

\section{INTRODUCTION}

The current Korean and Japanese guidelines recommend gastrectomy as the standard additional treatment after non-curative endoscopic submucosal dissection (ESD) for early gastric cancer (EGC). However, when the presence of positive lateral margins (pLMs) is the only noncurative factor, the risk of lymph node metastasis (LNM) is very low. ${ }^{1-3}$ Therefore, in such patients, additional ESD or argon plasma coagulation (APC) may be considered to avoid invasive surgeries. To date, however, only few studies have evaluated whether the long-term outcomes of additional ESD or APC are comparable to those of rescue surgery after non-curative ESD. In a recent study by Kim et al., ${ }^{4}$ no recurrence was observed among 23 patients with pLMs as the only non-curative factor who underwent additional ESD after non-curative resection. However, their study was limited due to short follow-up duration (mean, $12.7 \pm 12.5$ months). To guarantee the comparable outcomes of additional ESD or APC to surgery, a study with sufficient follow-up duration is necessary. In the present study, we aimed to elucidate the long-term outcomes of patients who underwent additional ESD or APC after non-curative ESD with pLMs as the only non-curative factor. 


\section{MATERIALS AND METHODS}

\section{Patients}

Between December 2001 and December 2016, 4,634 patients with 4,865 differentiated-type EGCs underwent their first ESD at Samsung Medical Center. Differentiatedtype EGCs included well- or moderately differentiated EGCs and papillary EGCs. Among these patients, 27 patients with 27 EGCs arising in the remnant stomach and five patients with five EGCs occurring in the reconstructed gastric tube after esophagectomy were excluded from the study population. Of the remaining patients, 909 patients with 920 EGCs underwent non-curative resection. We excluded 804 patients with 813 EGCs who had non-curative factors other than pLMs. Six patients with six EGCs who did not undergo additional treatment were also excluded. Finally, 99 patients with 101 EGCs with pLMs as the only non-curative factor were included in the analysis (Fig. 1).

Papillary adenocarcinoma was defined as a tumor with papillary structures composed of epithelial projections with a central fibrovascular core as a scaffold. ${ }^{5-8}$ Histological heterogeneity was defined when signet ringcell carcinoma or poorly differentiated adenocarcinoma components were found in less than $50 \%$ of the tumor area in an ESD specimen, in accordance with the Korean and Japanese guidelines. ${ }^{1,2}$

Clinicopathological data were obtained through the retrospective review of medical records from the intranet resources of Samsung Medical Center. We used Charlson comorbidity index to evaluate the comorbidity status. ${ }^{9}$ All enrolled patients provided written informed consent ac- cording to our institutional guidelines. The study protocol was approved by the Institutional Review Board of Samsung Medical Center (IRB number: 2018-08-143-002) and conducted in accordance with the guidelines of the Declaration of Helsinki.

\section{Initial ESD procedures and histopathological evaluation}

Preoperative chromoendoscopy using $0.2 \%$ indigo carmine was performed in all cases to delineate the tumor borders. ESD was performed by experienced endoscopists using standardized techniques and identical instruments. After ESD, the specimens were serially sectioned at 2-mm intervals and evaluated for tumor involvement in four lateral directions (distal, proximal, anterior, and posterior) and in the vertical direction. A detailed description of the ESD procedures and histopathological evaluation performed in our institution have been presented elsewhere. $^{10,11}$

\section{Additional treatments after non-curative resection}

The additional treatment modality was selected by the attending physician after evaluating the clinicopathological factors, including the final pathology report, age, the presence of underlying diseases, and consent to surgery. Subsequent ESD and histopathological evaluation procedures were performed in the same standard manner as the initial ESD. ${ }^{12}$ Additional APC was performed using an argon gas source with a high-frequency generator (Erbe Elektromedizin, Tübingen, Germany). The argon gas flow rate was $1.8 \mathrm{~L} / \mathrm{min}$, and the electrical current was

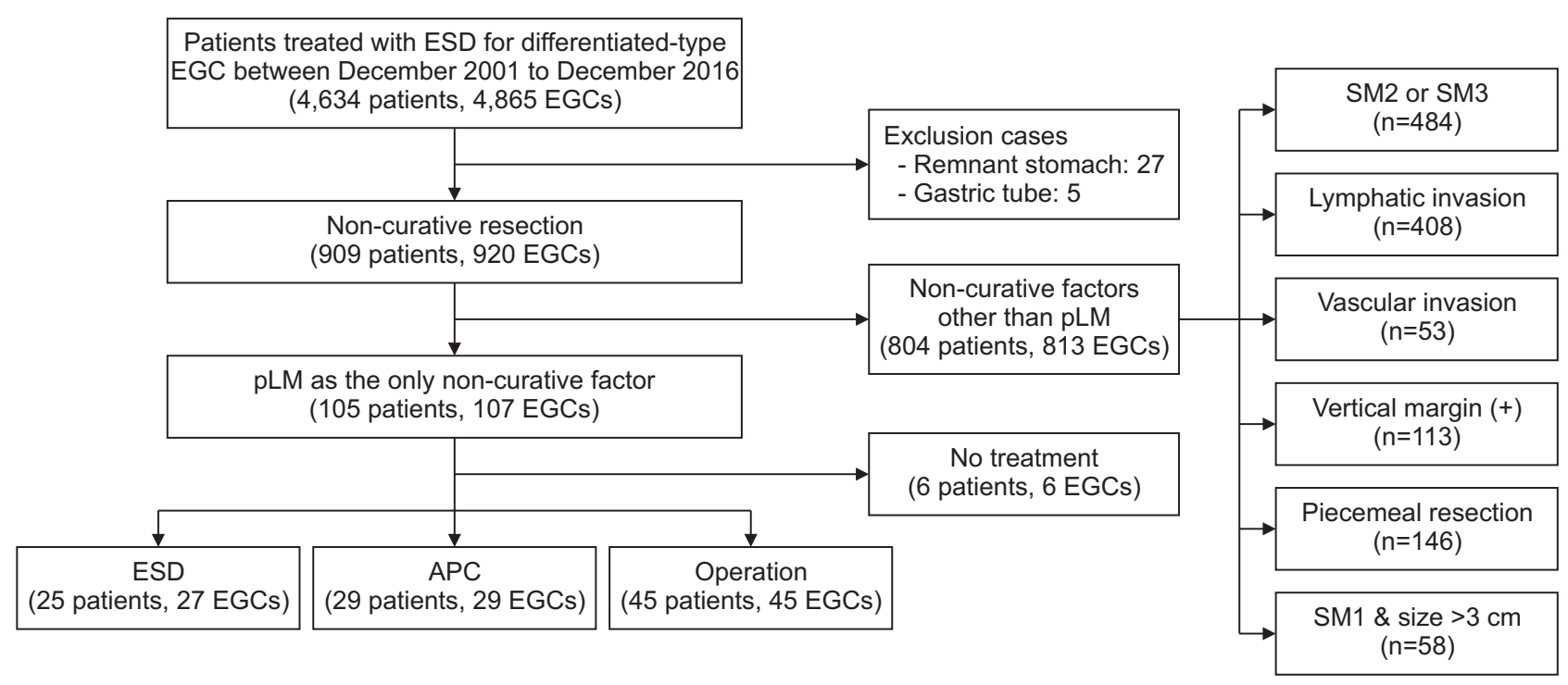

Fig. 1. Patient flowchart.

ESD, endoscopic submucosal dissection; EGC, early gastric cancer; pLM, positive lateral margin; APC, argon plasma coagulation; SM1, submucosal invasion depth $<500 \mu \mathrm{m}$ from the muscularis mucosa layer; SM2 or SM3, submucosal invasion depth $\geq 500 \mu \mathrm{m}$ from the muscularis mucosa layer. 
set at 30-50 W. In case the exact location and extent of the residual tumor was obscure, APC was done circumferentially along the margin of the post-ESD ulcer to ensure the complete ablation of residual tumor (Fig. 2). ${ }^{13}$

\section{Follow-up schedule}

Esophagogastroduodenoscopy (EGD) with biopsy was performed 2 months after ESD to confirm healing of the artificial ulcer and exclude any recurrence. Thereafter, EGD with biopsy and abdominal computed tomography were performed at 6-month intervals for 3 years, and then annually for the next 2 years. The follow-up duration for recurrence was defined as the time from ESD to the last follow-up date of EGD or computed tomography.

\section{Definitions}

Resection was defined as curative when a differentiated-type EGC underwent en bloc resection and showed negative lateral and vertical resection margins with no lymphovascular invasion, and fulfilled one of the following criteria: $^{2}$ (1) tumor size $\leq 2 \mathrm{~cm}$, mucosal cancer, no ulcer; (2) tumor size $>2 \mathrm{~cm}$, mucosal cancer, no ulcer; (3) tumor size $\leq 3 \mathrm{~cm}$, mucosal cancer, ulcer present; or (4) tumor size $\leq 3 \mathrm{~cm}$, SM1 cancer (submucosal invasion depth $<500 \mu \mathrm{m}$ from the muscularis mucosa layer). Non-curative ESD was defined when the curative resection criteria above was not met.

Residual lesion was defined as cancer detected at the ESD site within 12 months after ESD. When the cancer was detected at the ESD site after 12 months, it was defined as local recurrence. Metachronous recurrence was defined as a cancer detected at sites other than the ESD site during follow-up EGD, at least 12 months after ESD.

\section{Statistical analysis}

Categorical variables were analyzed using the chi-square test or the Fisher exact test. Continuous variables were analyzed using the Student t-test or the Mann-Whitney test. Statistical significance was set at $p<0.05$. All analyses were performed using SPSS version 25.0 (IBM Corp., Armonk, NY, USA).

\section{RESULTS}

\section{Clinicopathological characteristics of the patients}

The baseline clinicopathological characteristics of the 99 patients with 101 EGC lesions who underwent additional treatments after non-curative ESD are summarized in Table 1. Among the three treatment groups, there were no significant differences with respect to the comorbidity status and the proportion of patients taking antiplatelet or anticoagulation agents. There were $64(64.0 \%)$ cases with a single pLM and $36(36.0 \%)$ cases with multiple pLMs (one case in the APC group had no report on the multiplicity of involved margins). After additional ESD or surgery, residual tumors were found in $73.6 \%$ (53/72) of cases.

\section{Comparison of three additional treatments}

We compared the clinicopathological characteristics of patients undergoing three types of additional treatments (Table 1). Among the 101 cases with pLM as the only noncurative factor, 27 (26.7\%) underwent additional ESD, 29 (28.7\%) underwent additional APC, and 45 (44.6\%) underwent surgery. All additional endoscopic treatments were performed within three months after the initial ESD (ESD: median, 3 days; range, 1 to 13 days; APC: median, 3 days; range, 0 to 71 days). The median time interval between the initial ESD and additional surgery was 12 days (range, 1 to 3,660 days). Tumors with endoscopically depressed shape were most frequently found in the APC group (APC $65.5 \%$, ESD $33.4 \%$, and surgery $17.8 \%, \mathrm{p}=0.001$ ). Cases with multiple pLMs were most frequently found in the surgery group (surgery $53.3 \%$, APC $25.0 \%$, and ESD $18.5 \%$, $\mathrm{p}=0.004$ ). Patients in the APC group were older than those in the ESD and surgery groups (median age: 68, 59, and 60 years for the APC, ESD, and surgery groups, respectively; $\mathrm{p}=0.038$ ).
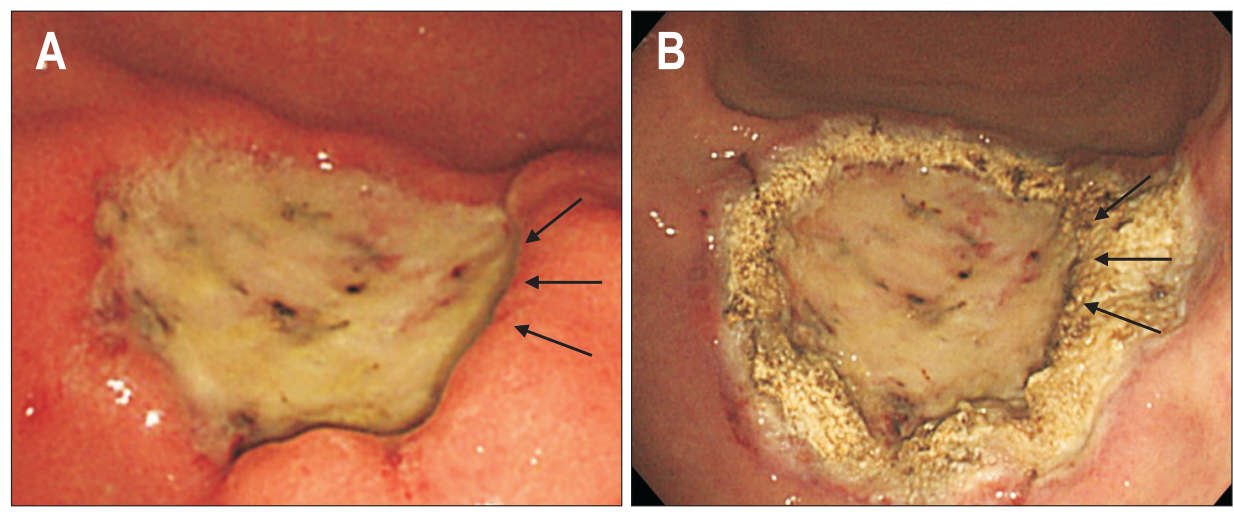

Fig. 2. A representative image of circumferential argon plasma coagulation in a patient with a positive posterior resection margin (arrows) after endoscopic submucosal dissection for early gastric cancer. As the extent of the residual tumor was obscure, argon plasma coagulation was performed circumferentially to ensure the complete ablation of residual tumor (A, B). 
Table 1. Comparison of Clinicopathological Characteristics among Patients with Positive Lateral Margins Undergoing Different Additional Treatments

\begin{tabular}{|c|c|c|c|c|c|}
\hline Characteristics & $\begin{array}{c}\text { Total } \\
(n=101)\end{array}$ & $\begin{array}{l}\text { Additional ESD } \\
\quad(n=27)\end{array}$ & $\begin{array}{c}\text { Additional APC } \\
\qquad(n=29)\end{array}$ & $\begin{array}{l}\text { Surgery } \\
(n=45)\end{array}$ & $\mathrm{p}$-value \\
\hline Age, yr* & & & & & 0.038 \\
\hline Mean \pm SD & $61.9 \pm 11.2$ & $60.8 \pm 12.6$ & $66.1 \pm 10.2$ & $59.9 \pm 10.5$ & \\
\hline Median (range) & $62(38-86)$ & $59(38-86)$ & $68(45-81)$ & $60(39-86)$ & \\
\hline Sex* & & & & & 0.602 \\
\hline Male & $67(67.7)$ & $18(72.0)$ & $21(72.4)$ & 28 (62.2) & \\
\hline Female & 32 (32.3) & $7(28.0)$ & $8(27.6)$ & 17 (37.8) & \\
\hline Hypertension* & & & & & 0.244 \\
\hline No & $60(60.6)$ & $17(68.0)$ & 14 (48.3) & $29(64.4)$ & \\
\hline Yes & $39(39.4)$ & 8 (32.0) & $15(51.7)$ & $16(35.6)$ & \\
\hline Charlson comorbidity index* & & & & & 0.267 \\
\hline 0 & $56(56.6)$ & $12(48.0)$ & $15(51.7)$ & $29(64.4)$ & \\
\hline 1 & $24(24.2)$ & $9(36.0)$ & 9 (31.0) & 6 (13.3) & \\
\hline 2 & $14(14.1)$ & $4(16.0)$ & $3(10.3)$ & $7(15.6)$ & \\
\hline 3 or more & $5(5.1)$ & 0 & $2(7.0)$ & $3(6.7)$ & \\
\hline Antiplatelet therapy or anticoagulation* & & & & & 0.485 \\
\hline No & 84 (84.8) & $21(84.0)$ & 23 (79.3) & 40 (88.9) & \\
\hline Yes & $15(15.2)$ & $4(16.0)$ & $6(20.7)$ & $5(11.1)$ & \\
\hline Tumor site & & & & & 0.887 \\
\hline Antrum/angle & $52(51.5)$ & $13(48.2)$ & $17(58.6)$ & 22 (48.9) & \\
\hline LB/MB & $32(31.7)$ & $10(37.0)$ & $8(27.6)$ & $14(31.1)$ & \\
\hline HB/fundus & $17(16.8)$ & $4(14.8)$ & $4(13.8)$ & $9(20.0)$ & \\
\hline Tumor shape & & & & & 0.001 \\
\hline Elevated & $37(36.6)$ & $10(37.0)$ & $7(24.1)$ & $20(44.4)$ & \\
\hline Flat & $28(27.7)$ & $8(29.6)$ & $3(10.4)$ & $17(37.8)$ & \\
\hline Depressed & $36(35.7)$ & $9(33.4)$ & 19 (65.5) & $8(17.8)$ & \\
\hline Tumor depth & & & & & 0.138 \\
\hline Mucosa & $95(94.1)$ & 26 (96.3) & $29(100.0)$ & 40 (88.9) & \\
\hline SM1 & $6(5.9)$ & $1(3.7)$ & 0 & $5(11.1)$ & \\
\hline Pathologic size, $\mathrm{cm}$ & & & & & 0.077 \\
\hline Mean $\pm S D$ & $2.8 \pm 1.4$ & $2.9 \pm 1.2$ & $2.3 \pm 1.2$ & $3.0 \pm 1.5$ & \\
\hline Median (range) & $2.6(0.4-7.8)$ & $3.0(0.6-5.4)$ & $2.2(0.4-5.4)$ & $2.8(0.9-7.8)$ & \\
\hline Tumor pathology & & & & & 0.355 \\
\hline WD & $37(36.6)$ & $8(29.6)$ & $12(41.4)$ & $17(37.8)$ & \\
\hline $\mathrm{MD}$ & $61(60.4)$ & $18(66.7)$ & $15(51.7)$ & $28(62.2)$ & \\
\hline Papillary & $3(3.0)$ & $1(3.7)$ & $2(6.9)$ & 0 & \\
\hline Histologic heterogeneity & & & & & 0.115 \\
\hline Absent & $81(80.2)$ & 23 (85.2) & 26 (89.7) & $32(71.1)$ & \\
\hline Present & 20 (19.8) & $4(14.8)$ & $3(10.3)$ & $13(28.9)$ & \\
\hline Margin involvement multiplicity ${ }^{+}$ & & & & & 0.004 \\
\hline Single & $64(64.0)$ & 22 (81.5) & $21(75.0)$ & $21(46.7)$ & \\
\hline Multiple & $36(36.0)$ & 5 (18.5) & $7(25.0)$ & 24 (53.3) & \\
\hline Residual tumor & & & & & 0.592 \\
\hline Absent & - & 6 (22.2) & - & 13 (28.9) & \\
\hline Present & - & $21(77.8)$ & - & $32(71.1)$ & \\
\hline Lymph node metastases & & & & & - \\
\hline Absent & - & - & - & 44 (97.8) & \\
\hline Present & - & - & - & $1(2.2)$ & \\
\hline Time to additional treatment, day & & & & & $<0.001$ \\
\hline Mean $\pm S D$ & $48.8 \pm 363.6$ & $4.1 \pm 3.2$ & $12.2 \pm 16.6$ & $99.1 \pm 543.6$ & \\
\hline Median (range) & $6(0-3,660)$ & $3(1-13)$ & $3(0-71)$ & $12(1-3,660)$ & \\
\hline
\end{tabular}

Data are presented as the number (\%) unless otherwise indicated.

ESD, endoscopic submucosal dissection; APC, argon plasma coagulation; LB, low body; MB, mid-body; HB, high-body; SM1, submucosal invasion depth $<500 \mu \mathrm{m}$ from the muscularis mucosa layer; WD, well-differentiated; MD, moderately differentiated.

${ }^{*}$ A total of 25 patients underwent ESD; ${ }^{\dagger}$ One case in the APC group with no report of margin involvement multiplicity was excluded. 
There were no complications such as bleeding or perforation among those undergoing additional APC or surgery. Among 27 attempts of additional ESD, no major bleeding occurred but three perforation cases were observed $(3 / 27$, $11.1 \%)$.

\section{Comparison of clinicopathological features of patients with and without residual tumor after additional treatment}

The comparison of clinicopathological features of patients with and without residual tumors after additional treatment for non-curative ESD with pLMs as the only non-curative factor is summarized in Table 2. Residual tumors were more frequently found in cases with multiple pLMs than in those with a single pLM.

\section{Long-term follow-up outcomes of three additional treatments}

Fig. 3 shows the follow-up outcomes after additional treatment in patients with pLMs as the only non-curative factor. The median follow-up duration for the entire study cohort was 58.9 months (range, 3.8 to 160.6 months). The

Table 2. Comparison of Clinicopathological Characteristics of Patients with and without Residual Tumors after Additional Treatment

\begin{tabular}{lccc}
\hline \multicolumn{1}{c}{ Characteristics } & $\begin{array}{c}\text { Residual } \\
\text { tumor }(n=53)\end{array}$ & $\begin{array}{c}\text { No residual } \\
\text { tumor }(n=19)\end{array}$ & p-value \\
\hline Tumor site & & & 0.493 \\
$\quad$ Antrum/angle & $26(49.1)$ & $9(47.4)$ & \\
LB/MB & $19(35.8)$ & $5(26.3)$ & \\
HB/fundus & $8(15.1)$ & $5(26.3)$ & \\
Tumor shape & & & 0.889 \\
$\quad$ Elevated & $23(43.4)$ & $7(36.8)$ & \\
Flat & $18(34.0)$ & $7(36.8)$ & \\
Depressed & $12(22.6)$ & $5(26.3)$ & \\
Tumor depth & & & 1.000 \\
Mucosa & $48(90.6)$ & $18(94.7)$ & \\
SM1 & $5(9.4)$ & $1(5.3)$ & \\
Pathologic size, cm & & & 0.947 \\
Mean \pm SD & $3.0 \pm 1.4$ & $2.9 \pm 1.3$ & \\
Median (range) & $2.8(0.9-7.8)$ & $2.8(0.6-5.2)$ & \\
Lateral margin involvement multiplicity & & 0.046 \\
Single & $28(52.8)$ & $15(78.9)$ & \\
Multiple & $25(47.2)$ & $4(21.1)$ & \\
Tumor pathology & & & 0.688 \\
WD & $17(32.1)$ & $8(42.1)$ & \\
MD & $35(66.0)$ & $11(57.9)$ & \\
Papillary & $1(1.9)$ & 0 & \\
Histologic heterogeneity & & & 0.531 \\
No & $39(73.6)$ & $16(84.2)$ & \\
Yes & $14(26.4)$ & $3(15.8)$ & \\
\hline & & & \\
\hline
\end{tabular}

Data are presented as the number (\%).

LB, low body; MB, mid-body; HB, high-body; SM1, submucosal invasion depth $<500 \mu \mathrm{m}$ from muscularis mucosa layer; WD, well-differentiated; MD, moderately differentiated. median follow-up duration was comparable between the ESD and surgery groups (62.8 and 60 months, respectively), but was shorter in the APC group (35.6 months, $\mathrm{p}=0.001$ ). During follow-up, there was one case of local recurrence after additional ESD and one case of local recurrence after additional APC (25.6 months and 16.1 months after initial ESD, respectively). Gastrectomy with LN dissection was performed for both cases, but no LNM was identified. In one patient, a residual lesion was detected 10 months after additional APC; the lesion was completely cured with another ESD. Three patients developed metachronous recurrences after additional APC. Among them, two patients were completely cured with another ESD and one patient was lost to follow-up. There was no extragastric recurrence in both types of endoscopic treatment groups during the follow-up period. LNM was identified in one patient $(2.2 \%, 1 / 45)$ among those who underwent additional surgery. This patient had moderately differentiated mucosal EGC lesion measuring $5.6 \mathrm{~cm}$ in the ESD specimen and showed histological heterogeneity (signet ring-cell carcinoma composed $20 \%$ of the tumor area).

\section{DISCUSSION}

pLM after ESD for EGC is problematic because it is a risk factor for residual tumor ${ }^{12,14,15}$ and local recurrence. ${ }^{15-17}$ While it is known that additional treatment can reduce the local recurrence rate, ${ }^{18}$ the choice of the optimal modality is not defined. Although surgery can definitively achieve complete tumor removal, several previous studies ${ }^{3,414,19}$ have suggested that owing to the extremely low risk of LNM, local endoscopic treatments may be sufficient in cases where pLM is the only non-curative factor. To date, however, there were only few studies with sufficient follow-up duration to ensure the comparable outcomes of additional endoscopic treatments to that of surgery. In the present study, the median follow-up duration in the additional ESD group exceeded 5 years (median, 62.8 months; range, 20.2 to 151.0 months). During follow-up, there were no extragastric recurrences or gastric cancer-related deaths after additional ESD. To the best of our knowledge, our study has the longest follow-up duration to evaluate the outcomes of additional endoscopic treatments in cases with pLMs as the only non-curative factor after ESD for EGC.

Kim et al. ${ }^{4}$ have reported that undifferentiated histology and multiple pLMs were associated with residual tumors after ESD. However, undifferentiated histology by itself carries a high risk of pLMs. ${ }^{20}$ Since multivariate analysis was not performed in the study by Kim et al., ${ }^{4}$ it was unclear if multiple pLMs were independently associ- 


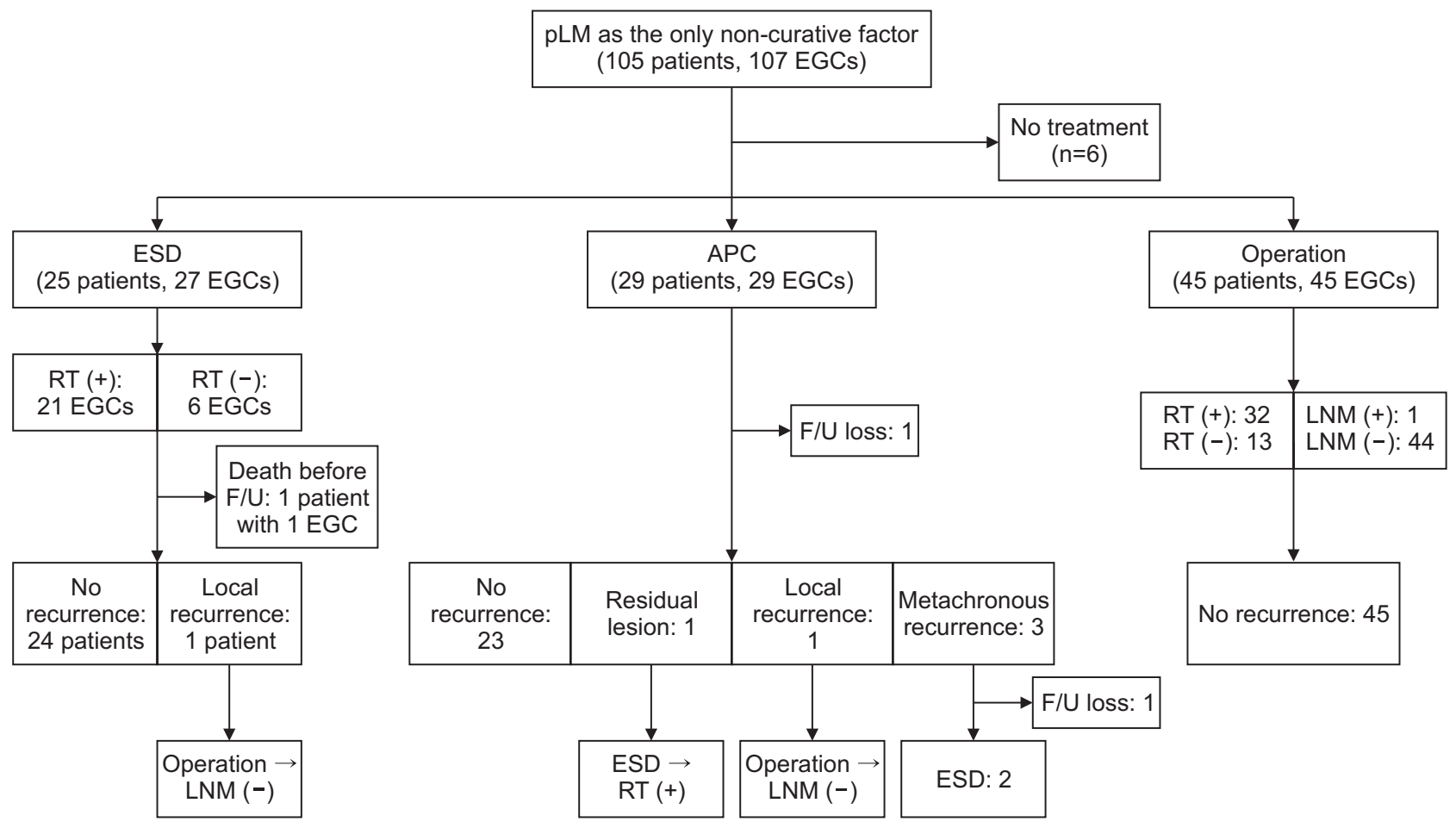

Fig. 3. Long-term outcomes of cases with pLMs as the only non-curative factor.

pLM, positive lateral margin; EGC, early gastric cancer; APC, argon plasma coagulation; ESD, endoscopic submucosal dissection; RT, residual tumor; LNM, lymph node metastases; F/U, follow-up.

ated with the presence of residual tumors, even in cases with differentiated-type EGCs. In the present study, which included only patients with differentiated-type EGCs, presence of multiple pLMs was found to be significantly associated with the presence of residual tumor (Table 2). This finding is consistent with that of previous studies, which have suggested that total length of tumor involvement in the margin was associated with residual or recurrent tumors. ${ }^{17,19,21}$ Based on these findings, it may be inferred that additional ESD may be preferable than APC in cases with multiple pLMs, considering that histological confirmation of complete removal of the residual tumor can be provided by ESD.

The efficacy and safety of APC on EGC treatment has been advocated in elderly or high-risk EGC patients ${ }^{13,22,23}$ because of its short operative time, ease of use, and lack of serious complications. ${ }^{24}$ The disadvantage of APC is that complete tumor eradication cannot be histologically confirmed. In the present study, there was no extragastric recurrence after APC during follow-up. There were no serious complications related to APC, such as bleeding or perforation. However, given the abovementioned limitation, careful follow-up is required after APC. In fact, residual or recurrent tumors were detected in two patients undergoing APC, which were curatively treated with ESD or surgery. In the present study, patients with depressed tumor shape were subjected to APC more frequently. We assume that this was probably influenced by the results of previous studies indicating that recurrences after APC were mostly detected in elevated-type tumors compared to depressed-type tumors, ${ }^{23-25}$ which was attributed to the greater tumor volume in elevated-type tumors than in depressed-type tumors of the same size.

The present and previous studies have shown favorable results for additional ESD or APC after non-curative ESD with pLMs as the only non-curative factor. In the present study, however, one patient with an EGC with histological heterogeneity was diagnosed with LNM postoperatively. EGC with histological heterogeneity is known for its aggressive clinicopathological behavior ${ }^{26,27}$ and high risk of LNM. Therefore, if the pathological assessment reveals histological heterogeneity and lateral margin involvement in the original ESD specimen, additional treatment methods should be very carefully selected between local endoscopic treatment and surgery.

In our previous study, no local recurrence was found after additional ESD or APC during a median follow-up of 38 months (range, 6 to 93 months). ${ }^{28}$ Kim et al. ${ }^{4}$ and Hoteya et $a{ }^{29}$ found no recurrences $(0 / 23$ and $0 / 11$, respectively) after additional endoscopic treatments (mean follow-up duration, $12.7 \pm 12.5$ months and $39.2 \pm 25.8$ months, respectively). In the present study, which included greater 
number of cases and had a longer follow-up duration than previous studies, the combined rate of residual lesions or local recurrences after additional ESD and APC was 4\% $(1 / 25)$ and $6.8 \%(2 / 29)$, respectively. However, there was no extragastric recurrence after additional endoscopic treatments during follow-up. All three patients with residual lesion or local recurrence after additional endoscopic treatments were completely cured by surgery or another ESD. No LNM was observed in these patients. Given these favorable long-term follow-up results and considering the impaired quality of life after gastrectomy, additional endoscopic treatment can be a reasonable choice for patients with pLMs as the only non-curative factor.

Still, complication risks need to be considered when choosing the method of additional treatment. In the present study, perforation occurred in $11.1 \%$ (3/27) of all additional ESD attempts. In one patient, ESD was stopped due to perforation during pre-cutting and was switched to APC (included in the APC group for long-term outcome analysis) after clipping. Another patient immediately underwent surgery (included in the surgery group for longterm outcome analysis). The other patient improved with supportive care only. It has been reported that degree of submucosal fibrosis is associated with higher rate of complications after ESD. ${ }^{30}$ Endoscopists should be aware that additional ESD might be more difficult and that it may carry higher risk of perforation compared to the initial ESD due to submucosal fibrosis.

This study has several limitations. This study had a retrospective design and was performed at a single tertiary referral center. As the number of cases was limited for each treatment modality, further large-scale studies are required. In addition, operator dependence on the treatment selection and procedural skills could not be adjusted due to the study design and limited number of cases. Also, it should be noted that the required time for getting the final pathology report after ESD varies between institutions. Consequently, the time interval between the initial ESD and the additional endoscopic treatment also varies between institutions. This difference in time interval can influence the degree of fibrosis and complication rates in the additional ESD. In our institution, the median time interval was only 3 days, and it might have led to favorable outcomes in the additional ESD. Although it is well known that additional ESD is feasible even in lesions with severe fibrosis, ${ }^{29,31}$ there can be high complication rates if the time interval between the initial ESD and the additional endoscopic treatment gets longer.

In conclusion, considering the comparable long-term outcomes of additional ESD or APC to those of surgery, additional ESD or APC may be considered an acceptable choice for patients with pLMs as the only non-curative factor after ESD for EGC.

\section{CONFLICTS OF INTEREST}

No potential conflict of interest relevant to this article was reported.

\section{AUTHOR CONTRIBUTIONS}

Conception and design: T.S.K., B.H.M. Analysis and interpretation of data: T.S.K., B.H.M. Acquisition of data: T.S.K., B.H.M., Y.W.M., H.L., P.L.R., J.J.K., J.H.L. Drafting of the article: T.S.K., B.H.M. Critical revision of the article for important intellectual content: T.S.K., B.H.M., Y.W.M., H.L., P.L.R., J.J.K., J.H.L. All authors have read and approved the final manuscript.

\section{ORCID}

Tae-Se Kim https://orcid.org/0000-0003-3950-4516 Byung-Hoon Min https://orcid.org/0000-0001-8048-361X Yang Won Min https://orcid.org/0000-0001-7471-1305 Hyuk Lee https://orcid.org/0000-0003-4271-7205 Poong-Lyul Rhee https://orcid.org/0000-0003-0495-5296 Jae J. Kim https://orcid.org/0000-0002-0226-1330 Jun Haeng Lee https://orcid.org/0000-0002-5272-1841

\section{REFERENCES}

1. Kim WH, Park CK, Kim YB, et al. A standardized pathology report for gastric cancer. Korean J Pathol 2005;39:106-113.

2. Japanese Gastric Cancer Association. Japanese gastric cancer treatment guidelines 2014 (ver. 4). Gastric Cancer 2017;20:119.

3. Oda I, Gotoda T, Sasako M, et al. Treatment strategy after non-curative endoscopic resection of early gastric cancer. $\mathrm{Br}$ J Surg 2008;95:1495-1500.

4. Kim HW, Kim JH, Park JC, et al. Additive endoscopic resection may be sufficient for patients with a positive lateral margin after endoscopic resection of early gastric cancer. Gastrointest Endosc 2017;86:849-856.

5. Kim TS, Min BH, Kim KM, Lee JH, Rhee PL, Kim JJ. Endoscopic submucosal dissection for papillary adenocarcinoma of the stomach: low curative resection rate but favorable long-term outcomes after curative resection. Gastric Cancer 2019;22:363-368 
6. Min BH, Byeon SJ, Lee JH, et al. Lymphovascular invasion and lymph node metastasis rates in papillary adenocarcinoma of the stomach: implications for endoscopic resection. Gastric Cancer 2018;21:680-688.

7. Sekiguchi M, Kushima R, Oda I, et al. Clinical significance of a papillary adenocarcinoma component in early gastric cancer: a single-center retrospective analysis of 628 surgically resected early gastric cancers. J Gastroenterol 2015;50:424434.

8. Lauwers GY, Carneiro F, Graham DY, Curado MP, Franceschi S, Montgomery E. Gastric carcinoma. In: Bosman FT, Carneiro F, Hruban RH, Theise ND, eds. WHO Classification of Tumours of the Digestive System. Lyon: IARC Press, 2010:48-58.

9. Charlson ME, Pompei P, Ales KL, MacKenzie CR. A new method of classifying prognostic comorbidity in longitudinal studies: development and validation. J Chronic Dis 1987;40: 373-383.

10. Min BH, Kim ER, Kim KM, et al. Surveillance strategy based on the incidence and patterns of recurrence after curative endoscopic submucosal dissection for early gastric cancer. Endoscopy 2015;47:784-793.

11. Pyo JH, Lee H, Min BH, et al. Long-term outcome of endoscopic resection vs. surgery for early gastric cancer: a noninferiority-matched cohort study. Am J Gastroenterol 2016; 111:240-249.

12. Bae SY, Jang TH, Min BH, et al. Early additional endoscopic submucosal dissection in patients with positive lateral resection margins after initial endoscopic submucosal dissection for early gastric cancer. Gastrointest Endosc 2012;75:432436.

13. Ahn JY, Choi KD, Na HK, et al. Clinical outcomes of argon plasma coagulation for the treatment of gastric neoplasm. Surg Endosc 2013;27:3146-3152.

14. Jung H, Bae JM, Choi MG, Noh JH, Sohn TS, Kim S. Surgical outcome after incomplete endoscopic submucosal dissection of gastric cancer. Br J Surg 2011;98:73-78.

15. Yoon H, Kim SG, Choi J, et al. Risk factors of residual or recurrent tumor in patients with a tumor-positive resection margin after endoscopic resection of early gastric cancer. Surg Endosc 2013;27:1561-1568.

16. Takenaka R, Kawahara Y, Okada H, et al. Risk factors associated with local recurrence of early gastric cancers after endoscopic submucosal dissection. Gastrointest Endosc 2008;68: 887-894.

17. Sekiguchi M, Suzuki H, Oda I, et al. Risk of recurrent gastric cancer after endoscopic resection with a positive lateral margin. Endoscopy 2014;46:273-278.

18. Jeon MY, Park JC, Hahn KY, Shin SK, Lee SK, Lee YC. Longterm outcomes after noncurative endoscopic resection of early gastric cancer: the optimal time for additional endo- scopic treatment. Gastrointest Endosc 2018;87:1003-1013.

19. Hwang JJ, Park KJ, Park YS, et al. A scoring system for patients with a tumor-positive lateral resection margin after endoscopic resection of early gastric cancer. Surg Endosc 2016;30:2751-2758.

20. Min BH, Kang KJ, Lee JH, et al. Endoscopic resection for undifferentiated early gastric cancer: focusing on histologic discrepancies between forceps biopsy-based and endoscopic resection specimen-based diagnosis. Dig Dis Sci 2014;59: 2536-2543.

21. Kim TK, Kim GH, Park DY, et al. Risk factors for local recurrence in patients with positive lateral resection margins after endoscopic submucosal dissection for early gastric cancer. Surg Endosc 2015;29:2891-2898.

22. Sagawa T, Takayama T, Oku T, et al. Argon plasma coagulation for successful treatment of early gastric cancer with intramucosal invasion. Gut 2003;52:334-339.

23. Tomita T, Arai E, Kohno T, et al. Outcomes of treatment of argon plasma coagulation therapy in elderly or high-risk patients with early gastric cancer: a comparison of outcomes among experienced and nonexperienced endoscopists. J Clin Gastroenterol 2011;45:e54-e59.

24. Kim KY, Jeon SW, Yang HM, et al. Clinical outcomes of argon plasma coagulation therapy for early gastric neoplasms. Clin Endosc 2015;48:147-151.

25. Kitamura T, Tanabe S, Koizumi W, Mitomi H, Saigenji K. Argon plasma coagulation for early gastric cancer: technique and outcome. Gastrointest Endosc 2006;63:48-54.

26. Min BH, Kim KM, Park CK, et al. Outcomes of endoscopic submucosal dissection for differentiated-type early gastric cancer with histological heterogeneity. Gastric Cancer 2015; 18:618-626.

27. Kim TS, Shin HC, Min BH, et al. Favorable long-term outcomes of endoscopic submucosal dissection for differentiated-type-predominant early gastric cancer with histological heterogeneity. J Clin Med 2020;9:1064.

28. Lee JH, Lee JH, Kim KM, Kang KJ, Min BH, Kim JJ. Clinicopathological factors of multiple lateral margin involvement after endoscopic submucosal dissection for early gastric cancer. Surg Endosc 2015;29:3460-3468.

29. Hoteya S, Iizuka T, Kikuchi D, et al. Secondary endoscopic submucosal dissection for residual or recurrent tumors after gastric endoscopic submucosal dissection. Gastric Cancer 2014;17:697-702.

30. Jeong JY, Oh YH, Yu YH, et al. Does submucosal fibrosis affect the results of endoscopic submucosal dissection of early gastric tumors? Gastrointest Endosc 2012;76:59-66.

31. Higashimaya M, Oka S, Tanaka S, et al. Endoscopic submucosal dissection for residual early gastric cancer after endoscopic submucosal dissection. Gastrointest Endosc 2013;77: 298-302. 